\title{
Automatic, Tensor-Guided Illustrative Vector Field Visualization
}

\author{
Cornelia Auer* \\ Zuse Institut Berlin
}

\author{
Jens Kasten ${ }^{\dagger}$ \\ University of Leipzig
}

\author{
Andrea Kratz \\ Zuse Institut Berlin
}

\author{
Eugene Zhang ${ }^{\S}$ \\ Oregon State University
}

\author{
Ingrid Hotz ${ }^{\mathbb{I}}$ \\ Zuse Institut Berlin
}

\begin{abstract}
This paper proposes a vector field visualization, which mimics a sketch-like representation. The visualization combines two major perspectives: Large scale trends based on a strongly simplified field as background visualization and a local visualization highlighting strongly expressed features at their exact position. Each component considers the vector field itself and its spatial derivatives. The derivate is an asymmetric tensor field, which allows the deduction of scalar quantities reflecting distinctive field properties like strength of rotation or shear. The basis of the background visualization is a vector and scalar clustering approach. The local features are defined as the extrema of the respective scalar fields. Applying scalar field topology provides a profound mathematical basis for the feature extraction. All design decisions are guided by the goal of generating a simple to read visualization. To demonstrate the effectiveness of our approach, we show results for three different data sets with different complexity and characteristics.
\end{abstract}

Index Terms: Computer Graphics [I.3.3]: Picture/Image Generation-Vector field visualization, illustrative visualization, asymmetric tensor fields, scalar field topology, homological persistence

\section{INTRODUCTION}

Visualization tries to embody much information of a data set within a single image. This often results in complex depictions that cannot be understood by domain experts without further knowledge of the visualization methods. As a remedy, illustrative visualization tries to mimic hand-drawn sketches $[6,34]$ of schematic illustrations as used by the domain experts themselves. In this paper, we present an automatic visualization tool similar to these hand drawn sketches.

Our major motivation is a sketch drawn by domain experts on the basis of a complex visualization [4] derived from an earthquake simulation [38]. Abstracting the complex original visualization results in a simple, easy-to-read image containing the most important information (Fig. 1). The goal of this work is to use computers to automatically generate similar abstracted, highly simplified representations of vector data and its prevalent features, pursuing the idea that showing less can be more. This can serve as a bridge towards other elaborate and more complete visualization methods and also eases the comparison of data sets, e.g., for different time steps.

Displaying less certainly requires a careful choice of what to show. This also implies that the abstraction process should only depend on a few intuitive parameters. Therefore, the design of our visualization is guided by the following ideas: generate a visualization that is simple in its concepts, intuitive in its single elements, conveys trends and strongly expressed features in the data.

\footnotetext{
*email: auer@zib.de

†email: kasten@informatik.uni-leizpig.de

¥email: kratz@zib.de

§email: zhange@eecs.oregonstate.edu

IIemail: hotz@zib.de
}

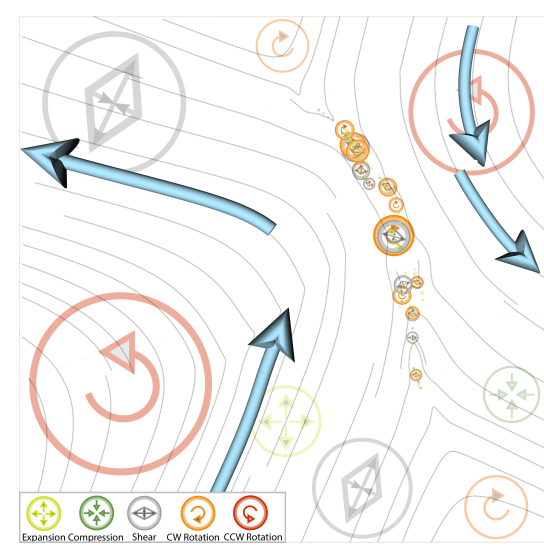

(a)

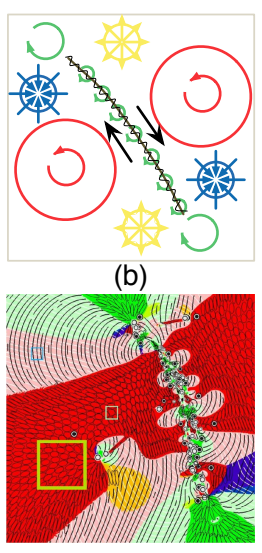

(c)
Figure 1: Visualization of a vector field from a simulation of coseismic displacements [38] (Sec. 5.2). The image (a) shows the result of our automatically generated visualization including a rough representation of the context as background and the strongly expressed features on top. Those occur mostly along the fault moving from top to down. The image (c) shows the detailed hybrid visualization technique developed by Chen et al. [4] consisting of hyperstreamlines and elliptical glyphs. Image (b) is the sketch drawn by domain experts on basis of (c) and motivated our work.

Visualization concept - The visualization consists of two major components: (a) a background visualization that displays large scale trends, and (b) a feature visualization that highlights locations of strongly expressed field characteristics. Both components are built from multiple layers considering the vector field itself and the gradient tensor field, an asymmetric tensor field. The derivative supplies valuable additional information about the vector field. The derivative, an asymmetric tensor, is decomposed according to Zhang et al. [40] into three components expressing the strengths of isotropic scaling, the rotation and the shear. In contrast to the work by Chen et al. [4], we do not display the entire tensor information but restrict ourselves to the magnitude of the components, which are scalar fields describing the respective feature strength.

The idea of the background visualization is to cluster regions of similar behavior. Thereby, only large scale clusters are considered for the visualization. The clustering method depends on the respective field. For vector fields, we introduce an efficient clustering approach, which is almost parameter free. For the clustering, we adopted the similarity measure proposed by Telea et al.[35] adding a component that considers the strength of the derived tensor field. As result, we obtain large clusters in mostly homogeneous regions while clusters are small in regions with high variations. For the scalar fields, we propose two approaches: the first delivers a clear partition of the domain into the dominant components of the scalar fields. However for its clarity it might loose important details. The second approach conveys more details and builds on scalar field topology exploiting basins of the maxima. To complement this trend visualization, we add a detailed feature visualization in the 
foreground. Local features are defined as maxima of the respective scalar field and offer the possibility to steer the level of detail for this representation via homological persistence. Features as well as background trends are represented by icons. The combination of these elements involves few plausible parameters which can be used to move the focus from global to detail or to specific features of interest.

In summary, the algorithm consists of the following components:

- Tensor decomposition

- Vector field clustering

- Scalar field topology extraction and persistence computation

- Cluster center computation for background glyph placement

- Rendering

The method is relevant for a multitude of application areas. We demonstrate its effectiveness using three data sets: A two dimensional flow behind a cylinder, a wind vector field from a multiparameter weather simulation and the displacement fields of an earthquake simulation.

\section{Related Work}

The analysis and visualization of vector fields has already a long tradition in the area of visualization. Accordingly, there has been done a large amount of interesting work in this field which is far beyond this paper. Considering different views on the topic, there are some good review articles: Texture and Feature-Based Flow Visualization [9], Topology-Based Flow Visualization [21], Partitionbased Techniques [32], and Illustrative Flow Visualization [2]. In relation to our work, especially, methods for vector field simplification and feature analysis are of interest.

Simplification via vector clustering - One approach to simplify complex data sets is to group regions of similar behaviors and represent them using on icon, e.g. one representative vector or a curved arrow icon. For all methods, the choice of an appropriate similarity measure on which the clustering is based is essential. Mostly, they are a combination of a position and vector (orientation and magnitude) error. Being able to display several levels of details the construction of vector field hierarchies has been the goal of several clustering algorithms. The methods can be mainly distinguished by choosing a top-down or bottom-up approach. An early work by Heckel et al. [12] recursively uses planes to split clusters based on streamline discrepancy as error measure. The result is a hierarchy of convex clusters. Another top-down approach using a generalized normalized-cut algorithm has been proposed by Chen et al. [5]. The goal of their method is to cluster vectors that are associated with the same critical point. Du et al. [7] propose a simplification of vector fields resulting in a predefined number of clusters based on Centroidal Voronoi tessellations (CVTs). Based on the chosen distance function, the vector fields are then naturally clustered by the CVT. A generalization of the K-means clustering has been introduced by Mc Kenzie et al. [23]. Its basic component is a variational clustering algorithm, that minimizes a global error for a given number of cluster sets. The algorithm is applicable to $2 \mathrm{D}$ and $3 \mathrm{D}$ fields and the error metric is flexibly exchangeable. There are also a couple of approaches pursuing a clustering based on an anisotropic diffusion process. In contrast to other clustering approaches, this is a continuous process without explicit split or merge events. Garcke et al. implemented a phase field model adapting the Cahn Hillard model to vector fields [10]. Greibel et al. propose to use an algebraic multi grid method to achieve similar results. While generating impressive results, theses methods do not provide a direct error control. The method we build on was developed by Telea et al. [35]. The goal of their work was to produce simplified but suggestive images without much user interaction. The clustering process works bottom-up starting with clusters for every data point. Then clusters are succes- sively merged according to differences in position and orientation and magnitude. A nice feature of the method is the design of the similarity measure which allows to control cluster shapes by adapting to the weights of the various terms in the error measure.

Simplification via streamline selection or clustering - Another approach is to simplify the vector field representation displaying only selected streamlines. There are several streamline placement algorithms with the goal to achieve a uniform coverage of the domain that is sparse but still gives a continuous impression of the field, e.g. for 2D fields [16, 29] and for 3D fields [22, 3]. Techniques that cluster precomputed streamlines are frequently used in context of fiber tracking for diffusion tensor data. An evaluation of related methods can be found in [24]. Kuhn et al. [20] have introduced a clustering technique using streamline properties, as curvature, to detect regions of similarity and visualize them by means of compact cluster boundaries.

A natural segmentation of vector fields in regions of similar streamline behavior is defined by its topological structure. Methods to simplify this structure and use it for visualization purposes have been proposed by Tricoche and Garth et al. [37, 11].

Feature extraction for vector fields - The second component of our visualization frame work builds on existing work on feature extraction and definition for vector fields. Papers in this area are concerned with the definition, stable extraction, and simplification of features. One can distinguish features that are directly defined on the vector vector field, as vector field topology, and features that facilitate a derived scalar field. Vector field topology thereby focuses on finding features like source, sinks, and saddle points as well as separatrices connecting them [21]. Scalar typed features are mostly defined as iso-contours for a given threshold or the extremal structure of the field [33]. Prominent examples are vortex like features defined on basis of vorticity [30,31], $\lambda_{2}$ [15], the Q quantity proposed by Hunt [14], or the acceleration magnitude [18].

An evolved analysis of multiple scalar and vector-like features has been presented by Zhang et al. [40, 4]. They consider the gradient vector field as basic feature carrier, which is composed in three components: isotropic scaling, rotation, and shear. While the first two components have only two distinct expressions, i.e., positive respective negative, the shear factor is also equipped with directional information. Based on this tensor decomposition, the domain is classified into regions where one feature dominates the others. We built on this work but facilitate the strength of the respective tensor components for a layered visualization. A decomposition with similar meaning is the Hodge decomposition which decomposes the field into a a divergence-free, a curl-free part, and a harmonic part. A topological analysis and multi-scale decompositions based on this decomposition has been persuaded in [27, 36]. A glyph-based visualization of time-dependent flows has been proposed in [13].

\section{BASICS AND NOTATION}

In the following let $\mathbf{v}$ be a $2 \mathrm{D}$ vector field given on the domain $D \in \mathbb{R}^{2} . \mathbf{v}: D \rightarrow \mathbb{R}^{2}$ maps a vector $\mathbf{v}(x)$ to each $x_{i} \in D$. T denotes an asymmetric tensor field representing the derivatives of $\mathbf{v}$.

\subsection{The gradient tensor field}

Besides the vector field itself its gradient (Jacobian) plays a fundamental role for the analysis of the field. Often it is referred to as a Galileian invariant entity which expresses shear, rotation and isotropic scaling of a fluid. In terms of a linear approximation of the field it appears as coefficient of the first term of the Taylor expansion. The linear approximation of $\mathbf{v}$ in point $a$ is given by

$$
\mathbf{v}_{1}(x)=\mathbf{v}(a)+\nabla \mathbf{v}(a)(x-a)
$$

$\mathbf{T}=\nabla \mathbf{v}$ defines an asymmetric tensor field over $D$. Transferring this to the task of vector field analysis and visualization leads to the two guiding ideas of our approach: 
1. Adding information given by the derivative helps to further/deeper understanding of the field, Section 3.2, 4.2, 4.3.3.

2. In locations where the derivative of $\mathbf{v}$ is small, the field is already well representend by the vectors given by $\mathbf{v}$, Section 4.3.2.

\subsection{Decompostion of derivative}

In the following the tensor derivative of $\mathbf{v}$ at a specific point in terms of an asymmetric tensor $T$ is represented by a $2 \times 2$ matrix $\left(T_{i j}\right)$. We pursue the decomposition of the tensor proposed by Zhang et al. [40] into independent components with clear physical meaning.

$$
\begin{aligned}
T & =D+S+R \\
& =\gamma_{d}\left(\begin{array}{ll}
1 & 1 \\
1 & 1
\end{array}\right)+\gamma_{s}\left(\begin{array}{cc}
\cos \theta & \sin \theta \\
\sin \theta & -\cos \theta
\end{array}\right)+\gamma_{r}\left(\begin{array}{cc}
0 & -1 \\
1 & 0
\end{array}\right)
\end{aligned}
$$

Without explicitly specifying the application area of the vector field $\mathbf{v}$ the components can be classified as follows: $D$ is the isotropic scaling, $S$ the shear, and $R$ the rotational part of $T$. $\theta$ gives the direction of angular deformation. The scalars $\gamma_{d}=\left(T_{11}+T_{22}\right) / 2$, $\gamma_{s}=\sqrt{\left(T_{11}-T_{22}\right)^{2}+\left(T_{12}+T_{21}\right)^{2}} / 2$, and $\gamma_{r}=\left(T_{21}-T_{12}\right) / 2$ are measurers for the strengths of $D, S$, and $R$ and allow to classify the vector field. Whilst the strength of the shear is always positive, $\gamma_{s}>0$, the sign of $\gamma_{r}$ and $\gamma_{d}$ distinguishes their quality.

With respect to the sign of these strength values we can specify five deformation modes:

- Positive isotropic scaling for $\gamma_{d}>0$

- Negative isotropic scaling for $\gamma_{d}<0$

- Pure shear with $\gamma_{s}>0$

- Counterclockwise rotation for $\gamma_{r}>0$

- Clockwise rotation for $\gamma_{r}<0$

The scalars $\gamma_{d}, \gamma_{r}$, and $\gamma_{s}$ and the tensor magnitude $m=\gamma_{d}^{2}+\gamma_{s}^{2}+\gamma_{r}^{2}$ will be used to guide and extend the vector field visualization as proposed above $(\operatorname{Sec} .3 .1 .1+2)$. In the following $\Gamma_{d}, \Gamma_{r}$, and $\Gamma_{s}$ denote the scalar fields to the strength values $\gamma_{d}, \gamma_{r}$, and $\gamma_{s}$.

\subsection{Scalar Field Topology}

For the background as well as the feature visualization we exploit concepts of scalar field topology. The features are defined as local extrema of the derived scalar fields $\Gamma_{d}, \Gamma_{s}$, and $\Gamma_{r}$ and the scalar field clustering uses basins of the extrema as fundamental structure (see also Fig. 10b). A basin of such a point is that part of the domain for which all points are connected with the respective extremum when integrating gradient lines. For instance, all points of a valley belong to the basin of its minimum. The basins of all minima present a segmentation that covers the whole domain. The same is true for the basins of all maxima.

To extract the scalar field topology we employ the combinatorial framework by Reininghaus et al. [28] which is robust and avoids the computation of further derivatives. Within this framework, we can use homological persistence as introduced by Edelsbrunner [8]. Homological persistence measures the stability of an extremal point against perturbation of the data values. It can be used to remove noise induced extremal points, but it also serves as a natural importance measure for critical points. An example is shown in Fig. 2, where the importance of the extremal points is determined by homological persistence. In this image, there are obviously outstanding extremal points, which are marked by high persistence values - homological persistence therefore provides a natural and sensible hierarchy of the extremal points. Selecting these exponents by mere thresholding could not provide such a well defined hierarchy and clarity in the image.

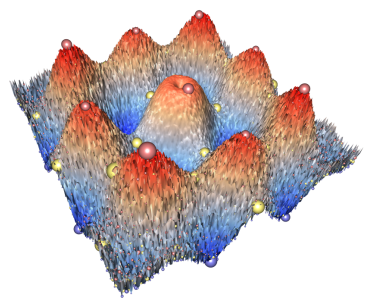

Figure 2: Critical points of a scalar function. The size of the spheres is determined by homological persistence. There are 38000 critical points contained in the dataset, but homological persistence classifies most of them as unimportant.

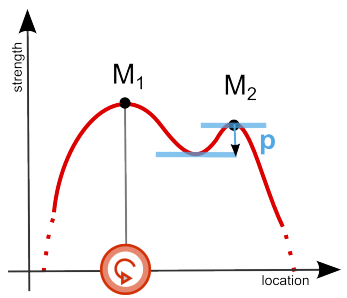

(a) low persistence

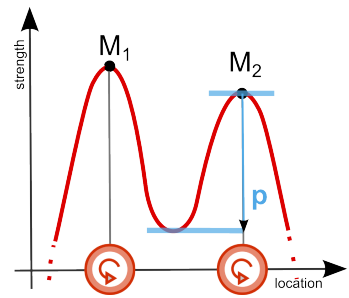

(b) high persistence
Figure 3: Simplified schematic illustration of the persistence concept. In both images $M_{1}, M_{2}$ are the maxima of a 1-D scalar function. In (a) $M_{2}$ has a relatively low, whereas in (b) $M_{2}$ has a high persistence value. Filtering by homological persistence would induce that for the function in (a) only at $M_{1}$ an icon would be drawn and the final depiction naturally cleaned up. In contrast, in (b) at both extrema, $M_{1}$ and $M_{2}$ an icon would be placed. However, we are convinced that although this might induce clutter both extrema are of such high importance that each of them has to be displayed.

For our application, we use homological persistence to control the level of detail for the represented feature points. We split the scalar fields $\Gamma_{s}, \Gamma_{r}$ which can also have negative values into two positive fields, respectively. Let $\Gamma_{i}, i \in\{d, r\}$ be the original scalar field. Then the two fields $\Gamma_{i+}, \Gamma_{i-}$ are defined as follows:

$$
\begin{gathered}
\Gamma_{i+}(x)= \begin{cases}\Gamma_{i}(x), & \Gamma_{i}(x)>0 \\
0, & \text { else }\end{cases} \\
\Gamma_{i-}(x)= \begin{cases}0, & \Gamma_{i}(x)>=0 \\
-\Gamma_{i}(x), & \text { else }\end{cases}
\end{gathered}
$$

Like this only maxima are extracted by the topology and persistence values unambiguously reflect their importance (see also Fig. 3).

\section{Visualization Concepts}

The essence of the visualization approach is to provide a composited image, where the most prominent features are presented in the foreground and in the background a context visualization is given.

The most prominent features are identified as the persistence filtered extrema of the scalar fields $\Gamma_{i}, i \in\{r+, r-, s, d+, d-\}$, (see Sec. 4.2). The background visualization comprises vector representation (see Fig. 4.3.2) and a rough simplified visualization of the quantities in the scalar fields $\Gamma_{i}$. To serve the different needs for understanding vector fields, we provide multiple strategies (see Sec. 4.3). Essentially they differ in the focus on either clarity of presentation or completeness of information. 


\subsection{Icons}

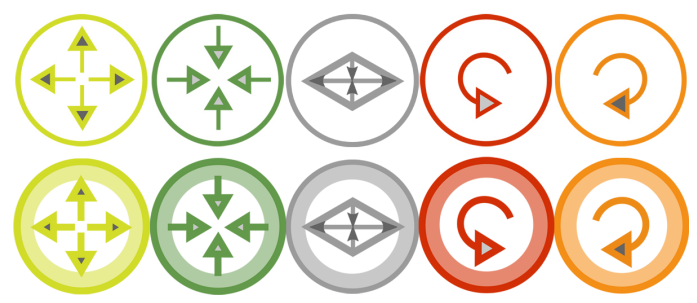

Figure 4: Top: Background icons for different deformation modes: positive, and negative isotropic scale, shear, counterclockwise rotation, and clockwise rotation. Bottom: Respective extrema icons.

Both extrema and background information deduced by the scalar fields are depicted by icons. The following goals were guiding their choice (Fig. 4): the icons should be simple to obtain a clear, harmonious image. Also the colors of the icons should be well discriminable, whilst avoiding too many colors in the image which might distract the spectator from the actual content. All icons are bounded by a circle, the base size of all icon is equal. The icons themselves only encode qualitative properties, the type of deformation mode. The isotropic scaling and rotation icons are rather self-explanatory. The shear icon qualitatively illustrates the deformation of a unit square under shear. The major arrow inside is oriented according to direction of the major eigenvector at the current location (the rhomb itself remains constant). The icons used for extrema are bounded by a bold circle to differentiate them from the background icons. Please note that the icons were designed that they could also work for color blind people.

\subsection{Extrema and foreground visualization}

As described in Sec. 3.3 features defined by the scalar fields $\Gamma_{i}, i \in$ $\{r+, r-, s, d+, d-\}$ are extracted as maxima of the scalar field topology. Filtering them by homological persistence allows to extract the most prominent features on a sound mathematical basis without corrupting the data (see also Fig. 5). Maxima due to topological noise are removed in a pre-processing step. The persistence value of the remaining extrema can be used to interactively determine the granularity of the representation. Note that the feature strength itself cannot be deduced from the persistence value.

To display the extracted features extremal-icons as introduced in Sec. 4.1 are placed at the location of the extrema. They are scaled according to their magnitude to encode the feature strength. Icons that fall below a predefined size (in our implementation one twentieth of the domain size) are depicted as colored dots.

\subsection{Background visualization}

For the background visualization of the vector data and the scalar data a clustering is performed. The resulting clusters are given as a region bonded by a polyline and are visualized using representative icons. The respective clustering algorithms, the definition and placement of the representatives are described in the following.

\subsubsection{Segment Voronoi diagram}

For the placement of the icons (arrows and background icons for the scalar fields) the segment Voronoi diagram by CGAL $[1,17]$ is employed which extracts the medial axis of the clusters. It takes line segments as input and supports non convex polygons and polygons with holes. Further it provides the minimum distance to the bounding polylines for each point on the medial axis. The use of the medial axis for the placement of the representatives is described in the respective sections.

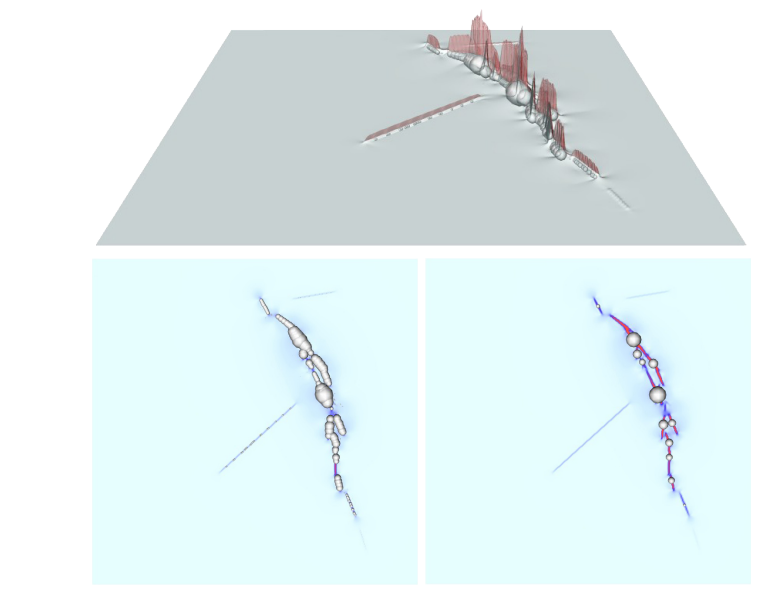

Figure 5: Earthquake data set (Sec. 5.2) a) Height field of shear scalar field. b) All extrema of scalar field displayed as spheres scaled by their persistence value. c) Persistence filtered extrema.

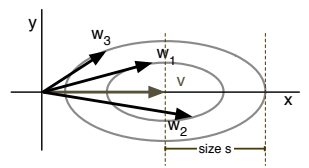

(a) directionError

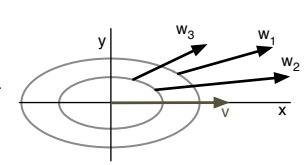

(b) positionError
Figure 6: (a) Isocontour errors as defined by [35].

\subsubsection{Vector field clustering and visualization}

For the purpose of generating a vector field clustering we decided to follow an approach similar to Telea et al. [35]. But in contrast to [35] we do not perform a hierarchical clustering of the complete field. Instead can we employ an efficient region growing method which starts in minimal values of the tensor magnitude. The method detailed below.

Choice of cluster region The aim of the vector clusters is to convey trends in the background. Therefore we restrict the clustering to regions where the tensor magnitude falls below a predefined threshold $\tau$ and the field is sufficiently represented by its vector value, Sec. 3.1.2. To respect the data set specific variations we set $\tau$ to a multiple of the maximal value $m_{\max }$ that the tensor magnitude reaches. In our implementation it is set to $\tau=0.01 \cdot m_{\max }$.

Similarity measure We adopt the anisotropic similarity measure for vectors of Telea et al. [35], which offers the option to trade-off between exact parallelism of vectors in the final clusters or clustering that follows streamlines.

We will shortly summarize the essential properties of the similarity measurement. For more detail please refer to the original paper [35]. In this paper an error space is introduced which combines feature and spatial space. Elliptically shaped iso-contours define similarity functions for vector directions and vector positions, see Fig. 6a, 6b. The eccentricity of a reference ellipse and its total size define the "strictness" of the similarity measure. The direction similarity compares the directions of two vectors which for extreme values of the reference ellipse eccentricity would return clusters of parallel vectors. The position similarity reflects how much the positions of the vectors is aligned with the vector field. For extreme ellipse eccentricity this would return clusters that are integrated streamlines. The final similarity function $e$ allows to trade- 
off between directional or position clustering the two error values $e_{d}$ and $e_{p}$ :

$$
e=(1-a) \cdot e_{d}+a \cdot e_{p}, a \in[0,1]
$$

All results in Sec. 5 where generated with an aspect ratio of the ellipses of $1: 2$ and $a=0.9$. A further variation compared to the method by Telea is that the size $s$ of the reference ellipse for the position error is set in relation to the mean magnitude of the tensor $m_{\text {mean }}$ (Eq. 5) inside the cluster, see Fig. 9 (c). This generically allows that large homogeneous regions are clustered even though the actual position distance becomes large.

Region growing As vectors are close to constant in the considered regions the clustering can be performed by a simple cell-based region growing algorithm. The clusters are stored as a list of cells and a bounding polyline. For the region growing a maximum error for the similarity measure (Eq. 4). is set to 0.01 . On demand this value can be changed by the user but was not necessary for all results in Sec. 5. The region growing starts in the cell with minimum tensor magnitude $m_{0}$, which is the location with the most homogeneous neighborhood. For the evaluation of the similarity function each cluster holds its mean vector, its center and the mean tensor magnitude $m_{\text {mean }}$. The size $s$ of the reference ellipse in the similarity function is set in relation to the mean magnitude of the cluster:

$$
s=\max \left(1, \log \left(1 / m_{\text {mean }}+1\right)\right)
$$

The clustering continues until adding a new cell would exceed the maximum error. In this case the clustering process recursively starts for a new cluster again in the (unclustered) cell with the minimum value of $m$. Cells with vectors close to zero are left out to avoid the clustering of singularities.

Visualization The extracted clusters are represented by a curved arrow icon. The arrow's tail is constituted by a streamline integrated within the cluster, and the head represents the direction of the vector field in that location. The start point for the streamline integration is located in the center of the medial axis (Sec. 4.3.1) of the clusters. It is defined as the center point on the longest path of the medial axis. From the start point an exact streamline integration [19] is performed in both directions (on $\mathbf{v}$ and $-\mathbf{v}$ ). For the display the arrows are shortened by $80 \%$.

\subsubsection{Scalar Quantities}

The background visualization of the scalar quantities is also based on clustering of regions of similar behavior. Thereby we offer two different approaches. The first is a fully automatic classification of the field characteristics with the advantage of a clear distribution of the representative icons in the image. However some small scale but strongly expressed components might be missing, therefore the second approach supports the depiction of the full information by offering an explorative understanding of the data.

\section{Classification - visualizing the dominant component}

To provide a first overview on the overall behavior in the field the classification into dominant components is performed according to see Chen et al. [4]. Here the relative ratios among the strengths $\gamma_{s}, \gamma_{d}$, and $\gamma_{r}$ are compared. By normalizing the strengths $\gamma_{s}^{2}+\gamma_{d}^{2}+\gamma_{r}^{2}=1$ all possible configurations can be modeled as a unit hemisphere. A Voronoi diagram on this hemisphere allows to classify the possible configurations to

- Isotropic scaling dominant $\mathbf{D}^{+}$(positive), $\mathbf{D}^{-}$(negative),

- Shear dominant $\mathbf{S}$,

- Rotation dominant $\mathbf{R}^{+}$(positive), $\mathbf{R}^{-}$(negative).

In our approach we exclude tensor magnitude values $m$ close to zero from the derivative visualization, to avoid the undefined

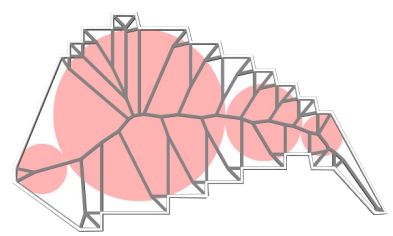

Figure 7: Placement of icons along medial axis of a cluster. The light gray polyline indicates the boundary of the cluster, the dark gray lines depict information by the segment Voronoi diagram (medial axis and distances to the boundary).

case, where the unit tensor is zero. Cells are labeled according to the dominant strength type and depicted according to the background visualization described below. The strongest advantage of this method is that the final image has a clean arrangement. However the drawback is that this classification does not consider the absolute feature strength. Further it is not possible to display regions where two characteristics play an important role.

\section{Basin visualization}

To offer a visualization that overcomes the limitations of an absolute classification this approach treats each scalar field separately. It uses the concept of topological basins as described in Sec. 3.3 which can be directly used as clusters for the icon placement. All icons are assigned the scalar value of their position which determines its transparency value in the final visualization. By changing a slider for a magnitude threshold the user can interactively blend in and out the icons of the regarded scalar field. The shear icons are additionally oriented in the direction of the major eigenvector. Please note that with the basin visualization background icons of different scalar fields may overlap, which is not the case for the classification approach.

\section{Icon placement}

All approaches for the background visualization (vectors and scalar fields) are technically treated equal. In a preprocessing step the boundaries of the extracted clusters are strongly simplified according to an angle criterion. For the simplified boundaries the medial axis is computed (Sec. 4.3.1). and the points on the medial axis are processed recursively. For the background icons of the scalar fields the placement starts in the point with the maximal distance to the boundary. Recursively we place the icons along the medial axis, by interpolating their optimal size by the distance values given at each point of the medial axis, see Figure 7.

\section{Results}

To verify the automatic generation of the sketch based we use three data sets with very different characteristics. All three data sets are inherently two-dimensional. For the two time-dependent data sets the analysis is performed on single time slices. For all data sets the same parameters are used.

Performance - our code basis has not been optimized towards performance yet. The most costly step is the persistence computation that is done for each scalar field (for details please refer to Reininghaus et al. [28]). For the data sets used in the results we denote the resolution and the processing time required for the topology computation for a single scalar field on a QuadCore i7 processor with 2,6 GHz. The computation time for the scalar fields is similar.

\subsection{Cylinder data set}

Data set - Time-dependent (32 time steps) two-dimensional CFD simulation of the von-Kármán vortex street [25, 39], the flow behind a cylinder with Reynolds number $=100$. The flow is incom- 


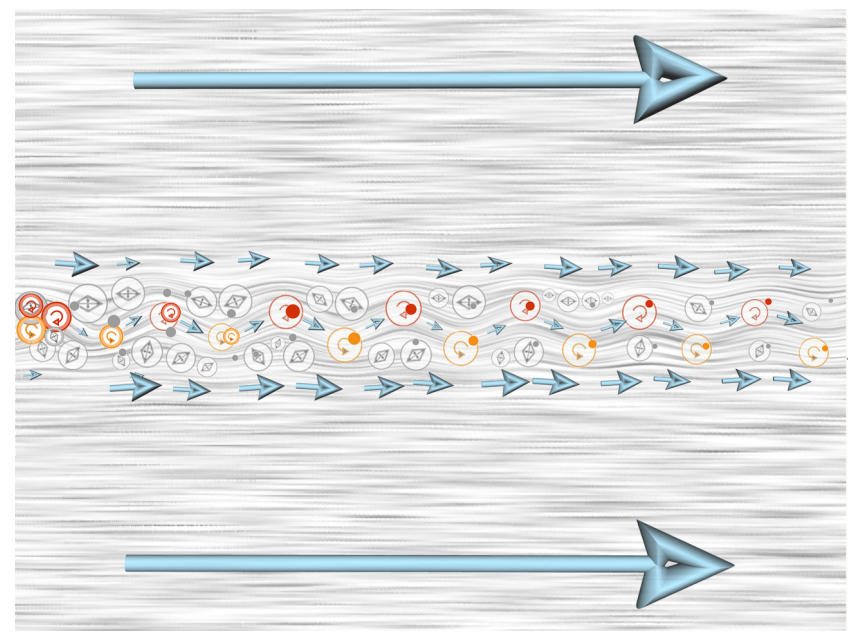

Figure 8: Cylinder data set - Information about shear and vorticity made visible in the sketch-like representation (in combination with the vector field visualization method LIC as context information). The extrema of the scalar fields show the locations of highest strengths. If the size of extrema is below the fixed threshold they are rendered as colored dots. The background visualization depicts the dominant components according to the classification: regions of dominant rotation are distinguished against those of dominant shear. Regions with low tensor magnitude are illustrated by arrows.

pressible, so by definition there is no isotropic scaling in the field. We use two versions of this data set: the original data and a version with changed reference frame with subtracted the average velocity. (Resolution: $242 \times 242$, Timing for scalar field topology: $0.47 \mathrm{sec}$ )

Analysis - Figure 8 and 9a, show all locations of strongest shear and rotation forces defined by the scalar field extrema. This is combined with the result of the classification into dominant components in the background. The image is further composited with the results of the vector clustering and a LIC visualization as context information. Traditional continuous vector visualization (i.e. LIC, or streamlines) does not encode direction nor velocity of the vectors. Thus important qualities like shear or vorticity might not be perceivable. Further a change of frame of reference might give a very different impression of the same vector field. This is demonstrated by Fig. 9a which could imitate the change of an observer's position. The LIC visualization in the background of Fig. 9a clearly differs from the one in Fig. 8. In contrast, the schematic illustrations deduced by the tensor decomposition remain independent of the frame of reference. In Fig. 9a the LIC might suggest strong vortical behavior throughout the flow from left to right. However the size of the extremal icons (or dots) clarifies that the magnitude of rotation at the right end is rather low. We consider it as an extension for the vector clustering methods described in Sec. 2 that the level of abstraction for the extrema has a clear mathematical reference and interpretation. This also supports the reproducibility of simplified and abstracted view on different data sets.

Figure $9 \mathrm{~b}$ illustrates the results of the vector clustering, clustered regions are represented by arrows. This image demonstrates how the clustering approach supports the generation of large clusters in homogeneous regions while still providing the necessary granularity in regions with higher variation. This sensitivity is a consequence of the adaptation of the position error of the similarity function to the underlying tensor magnitudes (Section 4.3.2) (see Fig. 9c). This would not be possible with a uniform measure as proposed in [35]. In context with flow analysis the mixing of flow is an interesting quality. For this purpose flow data sets are also inspected towards

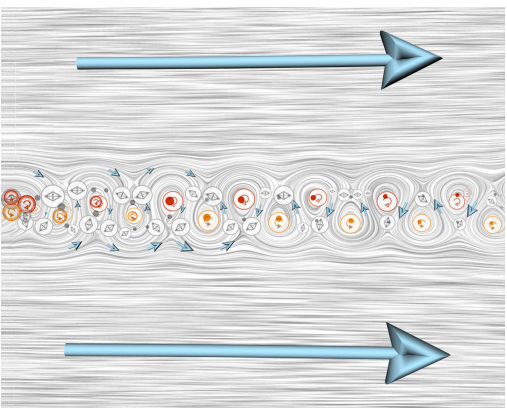

(a) (b)

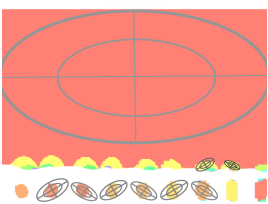

(c)

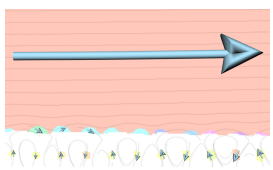

Figure 9: Cylinder data set - (a) Same data set as in Fig. 8 but with change of reference frame. Result shows the independence of the information given by the scalar fields from the chosen frame of reference. (b) Schematic depiction of the vector field clustering process with respect to magnitude of derivative (resulting clusters randomly color coded). White denotes regions with magnitude $m>\tau$, which are excluded from the clustering. (c) Close-up: schematic illustration of size adaption of the reference ellipse for position error.

its shear properties. After giving a first overview by the classified background visualization in Fig. 8 switching in the background to the basin visualization the overall behavior of the shear properties can be inspected. Fig. 10b exemplarily shows the extrema (dots with size scaled by persistence value) of the shear field and their respective basin regions.

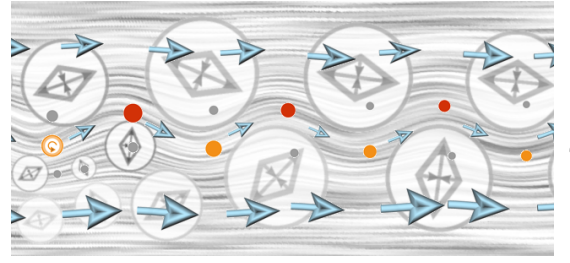

(a)

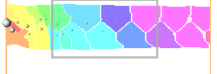

(b)
Figure 10: Cylinder data set - (a) Close-up: background visualization of shear basins (transparency of shear icons adjusted to strength, orientation to major eigenvector) for a more detailed inspection. The background visualization reveals properties of the shear which could not be deduced from the LIC visualization only. (b) Extrema (spheres with size adjusted to persistence value) and basins of shear scalar field. The gray rectangle denotes the close-up area in (a).

\subsection{Earthquake data set}

Data set - Displacement field encoding the ground deformation associated with a simulation of the June, $1992 \mathrm{Mw}=7.3$ Landers, CA earthquake [38].

(Resolution: $450 \times 450$, Timing for scalar field topology: $1.19 \mathrm{sec}$ )

Analysis - Figure 1a shows all extrema filtered by persistence (0.3) and scaled by magnitude. Among the extrema the location of the strongest forces can be distinguished by their size. The most prominent extrema align with the fault, where extrema of clockwise rotation and shear are dominant. The extrema of the isotropic scale only appear as colored dots. For the background visualization, the classification into dominant components according to Sec. 4.3 is done. This gives a very clear non overlapping image of the overall behavior in the field. The vector clustering captures the main directional features in the data set, additionally sparsely seeded streamlines are 
displayed in the background.

As one can see in the shear height field in Fig. 5a, the shear forces are very high in the vicinity of the fault, whereas they are comparatively small in the remainder of the data set. With the foreground visualization of the extrema icons, the attention is clearly drawn to the fault. The background visualization displays the trends in the surroundings which corresponds to a rather homogeneous but still relevant shear respectively rotational movement. This highly simplified depiction of strongest forces gives a clear first orientation, which can then be used in combination with a highly detailed visualization as in Fig. 1c. This visualization by Chen et al. focuses more on the directional information of the tensor components based on a classification. and does not offer such a clear and simple distinction of strong and weekly expressed features. Our resulting visualization nicely resembles the hand drawn sketch by domain experts [4], which felt the urge to deduce a strongly simplified schematic depiction of the most relevant features from the detailed visualization in Fig. 1c. Please note, in contrast to the hand drawn sketch, we also encode shear in the final visualization. Also the color coding of the icons differs.

\subsection{Climate data set}

Data set - Time dependent simulation of wind in a multi-model ensemble forecast system [26]. This simulation is freely available by the DEMETER project and aggregates different climate models with varying parameters. In our results, we inspect two consecutive time steps; images are computed separately. The data set is of low resolution with high variance and feature density.

(Resolution: $144 \times 73$, Timing for scalar field topology: $0.15 \mathrm{sec}$ )

Analysis - Here, we chose to compare the evolution of the wind data field and its associated characteristics by only inspecting the persistence filtered extrema (filtering factor: 0.3) and the vector clustering. As further context information sparsely seeded streamlines and the contours of the continents are provided. In the two time steps, related extrema can be visually well correlated due to the high level of abstraction by filtering and the simplicity of the icons (see shear icon in Fig. 11 gray square). Filtering by homological persistence does not avoid overlaps, but within a region of neighboring extrema the most persistent extremum serves as well defined representative. It provides a sound basis for stable and reproducible exponents in form of extrema to compare data over time, or according to varying models or parameters.

A detailed inspection of the climate data sets in combination with simple vector depiction demonstrates that the analysis of the extrema adds new information that cannot directly be deduced from the vector field. Figure 12 shows some close-ups of strong features, which revealed interesting patterns in the climate data set.

\section{Conclusion}

We proposed an illustrative visualization of two-dimensional vector fields consisting of two components: a background visualization that serves as an overview of large scale trends and a depiction of details in the foreground. The approach is based on the vector field and its first derivative, i.e., the vector gradient. We decomposed the gradient into shear, isotropic scaling and rotation and analyzed these individual fields. Our visualization pipeline therefore combines scalar field topology with tensor analysis. The highly abstracted view locates the foreground icons at positions with clear mathematical interpretation, the extrema of the scalar fields filtered by homological persistence. Homological persistence defines a hierarchy of importance for the extrema. This could not be accomplished by filtering the extrema by their magnitude only. The visualization is automatic. Only a few easy-to-handle parameters are used. In most cases, they can be set quite similar to default values as shown in the results. The final visualization incorporates

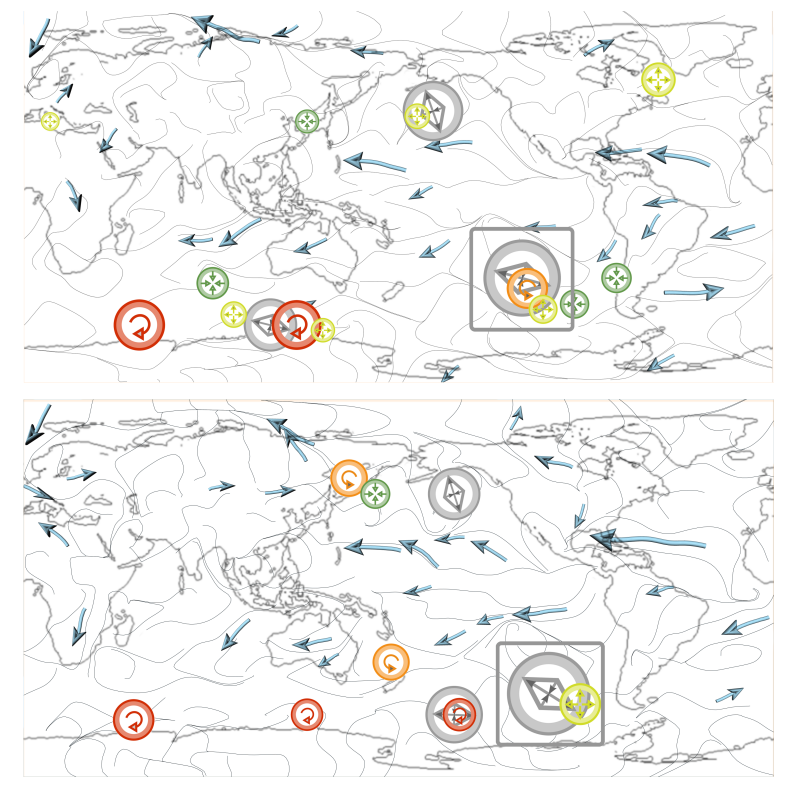

Figure 11: Climate data set - Time dependent simulation of wind. Top and bottom image display two consecutive time steps. Due to the high feature density the visualization is extremely simplified only the most persistent extrema are displayed. Additionally the arrow icons of the vector clustering, contours of the continents, and sparsely seeded streamlines can be seen.

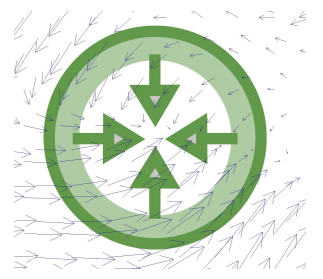

(a)

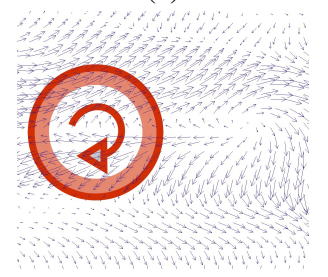

(c)

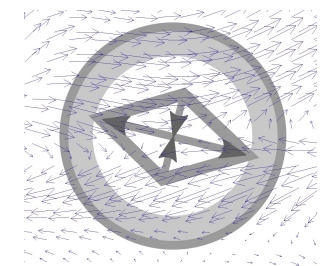

(b)

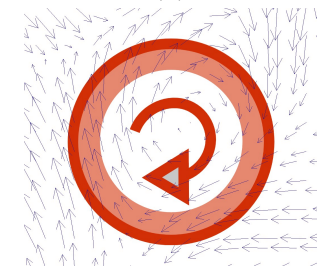

(d)
Figure 12: Climate data set - Close-ups of Fig. 11 (top row) combined with vector visualization. (a) Isotropic scaling extremum at location where the stream fronts converge from two sides which might indicate an up- or down-stream. (b) Shear extremum where the flow follows to opposing directions. $(c+d)$ The depicted scalar quantities are independent of the chosen frame of reference. In contrast to the extremum in (d) the rotation extremum in (c) is not in a location which the vector visualization would suggest.

quantities that might be hidden in pure vector visualization methods. In addition, it represents information that is independent of the chosen frame of reference. Therefore, it overcomes possible misinterpretations by only analyzing visualizations based on vectors. The icons are easy to interpret. The aim of this sketch-based visualization is not to provide all possible information in the vector field 
but rather to give an overview depiction. The resulting sketches are rather sparse and can be composited with more specific visualization methods for in-depth analysis. As future work, we plan to analyze time-dependent fields. The present approach directly allows the application of tracking methods for scalar field topology. This should result in smooth illustrative visualizations of these fields.

\section{ACKNOWLEDGEMENTS}

This work was partially supported by the

- DFG Emmy Noether Program (I. Hotz, C. Auer, A. Kratz).

- European Social Fund/ App.No.100098251 (J. Kasten)

- NSF award IIS-0917308 (E. Zhang).

\section{References}

[1] Cgal: Comp geom algo library. http://www.cgal.org

[2] A. Brambilla, R. Carnecky, R. Peikert, I. Viola, and H. Hauser. Illustrative Flow Visualization: State of the Art, Trends and Challenges. Eurographics STAR Reports, pages 75-94, 2012.

[3] C.-K. Chen, S. Yan, H. Yu, N. Max, and K.-L. Masahiro. An Illustrative Visualization Framework for 3D Vector Fields. Computer Graphics Forum, 30(7):1941-1951, 2011.

[4] G. Chen, D. Palke, Z. Lin, H. Ye, P. Vincent, R. S. Laramee, and E. Zhang. Asymmetric Tensor Field Visualization for Surfaces. IEEE Trans. on Visualization and Computer Graphics, 17(11):1979-1988, 2011.

[5] J.-L. Chen, Z. Bai, B. Hamann, and T. J. Ligocki. A Normalizedcut Algorithm for Hierarchical Vector Field Data Segmentation. In In Proc of Visualization and Data Analysis, 2003.

[6] U. Dallmann. Topological Structures of Three-Dimensional Flow Separations. Dfvlr-ava bericht nr. 221-82 a 07, DLR, Germany, 1983.

[7] Q. Du and D. Wang. Anisotropic Centroidal Voronoi Tessellations and Their Applications. SIAM J. on Scientific Computing, 26(3):737-761, 2005.

[8] H. Edelsbrunner, D. Letscher, and A. Zomorodian. Topological Persistence and Simplification. Discrete Comput. Geom., 28:511-533, 2002.

[9] G. Erlebacher, C. Garth, R. S. Laramee, H. Theisel, X. Tricoche, T. Weinkauf, and D. Weiskopf. Texture and Feature-Based Flow Visualization - Methodology and Application. In IEEE Visualization 06 Tutorial, 2006.

[10] H. Garcke, T. Preus̈er, M. Rumpf, A. C. Telea, U. Weikard, and J. J. van Wijk. A Phase Field Model for Continuous Clustering on Vector Fields. IEEE Trans. on Visualization and Computer Graphics, 7(3):230-241, 2001.

[11] C. Garth, X. Tricoche, T. Salzbrunn, T. Bobach, and G. Scheuermann. Surface Techniques for Vortex Visualization. In Data Visualization 2004 - TCVG Symp on Visualization, pages 155-164, 2004.

[12] B. Heckel, G. H. Weber, B. Hamann, and K. I. Joy. Construction of Vector Field Hierarchies. In Proc of IEEE Visualization, pages 19-26, 1999.

[13] M. Hlawatsch, P. Leube, W. Nowak, and D. Weiskopf. Flow radar glyphs - static visualization of unsteady flow with uncertainty. IEEE Trans. on Visualization and Computer Graphics, 17(12):1949-1958, 2011.

[14] J. C. R. Hunt. Vorticity and Vortex Dynamics in Complex Turbulent Flows. Trans. of the Canadian Society of Mechnical Engineering, 11:21-35, 1987.

[15] J. Jeong and F. Hussain. On the Identification of a Vortex. J. of Fluid Mechanics, 285:69-94, 1995.

[16] B. Jobard and W. Lefer. Multiresolution Flow Visualization. In Proc or WSCG, pages 33-37, 2001.

[17] M. I. Karavelas. A Robust and Efficient Implementation for the Segment Voronoi Diagrams. In Internat. Symp. on Voronoi diagrams in Science and Engineering (VD'04), pages 51-62, 2004.

[18] J. Kasten, J. Reininghaus, I. Hotz, and H.-C. Hege. Two-dimensional Time-dependent Vortex Regions based on the Acceleration Magnitude. IEEE Trans. on Visualization and Computer Graphics, 17(12):2080-2087, 2011.
[19] P. Kipfer, F. Reck, and G. Greiner. G.: Local Exact Particle Tracing on Unstructured Grids. Computer Graphics Forum, 22:133-142, 2003.

[20] A. Kuhn, D. J. Lehmann, R. Gaststeiger, M. Neugebauer, B. Preim, and H. Theisel. A Clustering-based Visualization Technique to Emphasize Meaningful Regions of Vector Fields. In Proc of Vision, Modeling, and Visualization (VMV'11), pages 191-198, 2011.

[21] R. S. Laramee, H. Hauser, L. Zhao, and F. H. Post. Topology-Based Flow Visualization, The State of the Art. In Topology-based Methods in Visualization, pages 1-19, 2007.

[22] S. Marchesin, C.-K. Chen, C. Ho, and K.-L. Ma. View-Dependent Streamlines for 3D Vector Fields. IEEE Trans. on Visualization and Computer Graphics, 16(6):1578-1586, 2010.

[23] A. McKenzie, S. V. Ombeyda, and M. Desbrun. Vector Field Analysis and Visualization through Variational Clustering. In Proc of The Symposium on Visualization, 2005.

[24] B. Moberts, A. Vilanov, and J. van Wijk. Evaluation of Fiber Clustering Methods for Diffusion Tensor Imaging. In Proceedings of IEEE Visualization Conference, pages 65-72, 2005.

[25] B. R. Noack, M. Schlegel, B. Ahlborn, G. Mutschke, M. Morzyński, P. Comte, and G. Tadmor. A Finite-time Thermodynamics of Unsteady Fluid Flows. J. Non-Equilibr. Thermodyn., 33(2):103-148, 2008.

[26] T. Palmer, A. Alessandri, U. Andersen, P. Cantelaube, M. Davey, P. Dlcluse, M. Dqu, E. Dez, F. Doblas-Reyes, and H. Feddersen. Development of a European multi-model ensemble system for seasonal to inter-annual prediction (DEMETER). Bulletin of the American Meteorological Society, 85(6):853-872, 2004.

[27] K. Polthier and E. Preuss. Identifying Vector Field Singularities Using a Discrete Hodge Decomposition. In Visualization and Mathematics III, pages 113-134. Springer, 2003.

[28] J. Reininghaus, D. Günther, I. Hotz, S. Prohaska, and H.-C. Hege. TADD: A Computational Framework for Data Analysis Using Discrete Morse Theory. In Mathematical Software - ICMS 2010, volume 6327, pages 198-208. Springer, 2010.

[29] O. Rosanwo, C. Petz, I. Hotz, S. Prohaska, and H.-C. Hege. Dual Streamline Seeding. In IEEE Pacific Visualization Symposium, pages 9-16, 2009.

[30] F. Sadlo, R. Peikert, and E. Parkinson. Vorticity Based Flow Analysis and Visualization for Pelton Turbine Design Optimization. In VIS '04: Proc of the conference on Visualization '04, pages 179-186, 2004.

[31] J. Sahner, T. Weinkauf, N. Teuber, and H.-C. Hege. Vortex and Strain Skeletons in Eulerian and Lagrangian Frames. IEEE Trans. on Visualization and Computer Graphics, 13(5):980-990, 2007.

[32] T. Salzbrunn, H. Jänicke, T. Wischgoll, and G. Scheuermann. The State of the Art in Flow Visualization: Partition-based Techniques. In Simulation and Visualization 2008 Proceedings, 2008.

[33] D. Schneider, A. Wiebel, H. Carr, M. Hlawitschka, and G. Scheuermann. Interactive Comparison of Scalar Fields Based on Largest Contours with Applications to Flow Visualization. IEEE Trans. on Visualization and Computer Graphics, 14(6):1475-1482, 2008.

[34] D. Schroeder, D. Coffey, and D. F. Keefe. Drawing with the flow: a sketch-based interface for illustrative visualization of $2 \mathrm{D}$ vector fields. In Proc of ACM SIGGRAPH/EG Sketch-Based Interfaces and Modeling, pages 49-56, 2010.

[35] A. Telea and J. J. van Wijk. Simplified Representation of Vector Fields. In VIS '99: Proceedings of the Conference on Visualization '99, pages 35-42, 1999.

[36] Y. Tong, S. Lombeyda, A. N. Hirani, and M. Desbrun. Discrete Multiscale Vector Field Decomposition. ACM Trans. on Graphics (TOG) (Siggraph'03), 22(3):445-452, 2003.

[37] X. Tricoche, G. Scheuermann, and H. Hagen. Continuous Topology Simplification of Planar Vector Fields. In VIS '01: Proceedings of the conference on Visualization '01, pages 159-166, 2001.

[38] P. Vincent. Application of SAR Interferometry to Low-rate Crustal Deformation Fields. PhD thesis, University of Colorado, 1998.

[39] C. Williamson. Vortex Dynamics in the Cylinder Wake. Annu. Rev. Fluid Mech., 28:477-539, 1996.

[40] E. Zhang, H. Yeh, Z. Lin, and R. S. Laramee. Asymmetric Tensor Analysis for Flow Visualization. IEEE Trans. on Visualization and Computer Graphics, 15(1):106-122, 2009. 